\title{
Role of Atg5-dependent cell death in the embryonic development of Bax/Bak double-knockout mice
}

\author{
Satoko Arakawa', Masatsune Tsujioka', Tatsushi Yoshida", Hajime Tajima-Sakurai ${ }^{1}$, Yuya Nishida', Yosuke Matsuoka, Ikuyo Yoshino ${ }^{1}$, \\ Yoshihide Tsujimoto ${ }^{*, 2}$ and Shigeomi Shimizu ${ }^{*, 1}$
}

Programmed cell death, which is required for the development and homeostasis of metazoans, includes mechanisms such as apoptosis, autophagic cell death, and necrotic (or type III) death. Members of the Bcl2 family regulate apoptosis, among which Bax and Bak act as a mitochondrial gateway. Although embryonic fibroblasts from Bax/Bak double-knockout (DKO) mice are resistant to apoptosis, we previously demonstrated that these cells die through an autophagy-dependent mechanism in response to various types of cellular stressors. To determine the physiological role of autophagy-dependent cell death, we generated Atg5/Bax/Bak triple-knockout (TKO) mice, in which autophagy is greatly suppressed compared with DKO mice. Embryonic fibroblasts and thymocytes from TKO mice underwent autophagy much less frequently, and their viability was much higher than DKO cells in the presence of certain cellular stressors, providing genetic evidence that DKO cells undergo Atg5-dependent death. Compared with wild-type embryos, the loss of interdigital webs was significantly delayed in DKO embryos and was even further delayed in TKO embryos. Brain malformation is a distinct feature observed in DKO embryos on the 129 genetic background, but not in those on a B6 background, whereas such malformations appeared in TKO embryos even on a B6 background. Taken together, our data suggest that Atg5-dependent cell death contributes to the embryonic development of DKO mice, implying that autophagy compensates for the deficiency in apoptosis.

Cell Death and Differentiation (2017) 24, 1598-1608; doi:10.1038/cdd.2017.84; published online 2 June 2017

Programmed cell death $(\mathrm{PCD})$ is a genetically regulated mechanism that is essential for diverse biological events such as morphogenesis and the elimination of harmful cells. ${ }^{1}$ Apoptosis is the major physiological mechanism mediating PCD, and is regulated by members of the Bcl2 family. ${ }^{2}$ However, PCD is also mediated by other mechanisms, such as autophagic cell death or type II PCD. ${ }^{3,4}$

Macroautophagy (hereafter referred to as autophagy) is an intrinsic cellular process that digests cellular components. Autophagy occurs constitutively at low basal levels and is accelerated by diverse cellular stressors. This machinery is driven by at least 30 autophagy-related proteins (Atgs) that are highly conserved from yeast to mammals. ${ }^{5,6}$ Evidence indicates that among these molecules, Atg5 and Atg7 are essential for autophagy. ${ }^{6}$ But we previously discovered that in addition to Atg5/Atg7-dependent autophagy, there an Atg5/ Atg7-independent mechanism of autophagy exists in mammalian cells. ${ }^{7}$

Autophagy is primarily a prosurvival mechanism. Therefore, it is frequently activated to suppress cell damage, when cells are subjected to severe stress. However, evidence suggests that autophagy contributes to cell death under certain conditions, ${ }^{8}$ and is therefore sometimes referred to as 'autophagic cell death'. In this context, this term is used only when nonapoptotic cell death occurs via the activation of autophagy, and cell death is rescued by the suppression of autophagy. ${ }^{9}$ In Drosophila, the programmed death of larval salivary gland cells is proposed to be a good example of autophagic cell death because ecdysone acts to remove the salivary glands via the transcriptional upregulation of autophagy genes. ${ }^{10,11}$ Furthermore, the genetic ablation of autophagy genes in Drosophila delays the elimination of salivary glands. Despite findings that the effects of autophagy genes are partial and that the apoptotic machinery appears to contribute to some extent, the PCD of salivary glands is described as autophagic cell death.

In mammals, we previously demonstrated that embryonic fibroblasts from Bax/Bak double-knockout (DKO) mice, in which the apoptosis machinery is blocked, die containing numerous autophagic structures after exposure to a variety of apoptotic stimuli. Furthermore, this type of cell death occurs through autophagy because it is prevented by autophagy inhibitors or by silencing of the expression of autophagy genes. ${ }^{12}$ Moreover, accumulating evidence indicates that autophagic cells are committed to undergo cell death under certain conditions. ${ }^{13}$ Although a large body of evidence suggests that mammalian cells undergo autophagic cell death, such data were acquired mainly from studies on cultured cells, and hence there is only limited evidence demonstrating autophagic cell death in vivo. To obtain genetic evidence of the occurrence of autophagic cell death during development or in the stress responses of mammals in vivo, we generated Atg5/Bax/Bak triple-knockout (TKO) mice and compared their phenotypes with those of Bax/Bak DKO mice

\footnotetext{
${ }^{1}$ Department of Pathological Cell Biology, Medical Research Institute, Tokyo Medical and Dental University, 1-5-45 Yushima, Bunkyo-ku, Tokyo 113-8510, Japan and ${ }^{2}$ Department of Molecular and Cellular Biology, Research Institute of Osaka Medical Center for Cancer and Cardiovascular Diseases, 1-3-2 Nakamichi, Higashinari, Osaka 537-8511, Japan

${ }^{*}$ Corresponding author: Y Tsujimoto or S Shimizu, Department of Pathological Cell Biology, Tokyo Medical and Dental University, Medical Reseach Institute, 1-5-45 Yushima, Bunkyo-ku, Tokyo 113-8510, Japan. Tel: +81 35803 4692; Fax: +81 35803 4821; E-mail: tsujimoto@mc.pref.osaka.jp or shimizu.pcb@mri.tmd.ac.jp Received 16.6.15; revised 18.3.17; accepted 27.4.17; Edited by A Villunger; published online 02.6.17
} 
because autophagic cell death is readily observed in apoptosis-resistant cells. The data presented here suggest that autophagic cell death contributes to the normal formation of the interdigital web and may be involved in embryonic viability and brain development in apoptosis-deficient mice, implying that autophagic cell death contributes to development by compensating for a deficiency in apoptosis.

\section{Results}

Generation of Atg5/Bax/Bak TKO mice. We previously reported that mouse embryonic fibroblasts (MEFs) from Bax/ Bak DKO mice die by autophagic cell death after exposure to various stressors. ${ }^{12}$ However, to our knowledge, there are no published studies that present genetic evidence of mammalian autophagic cell death in vivo. Therefore, we generated Atg5/Bax/Bak TKO embryos by breeding $\mathrm{Bax}^{+/-} \mathrm{Bak}^{-/-}$mice with ${\text { Atg } 5^{+/-} \text {mice. }}^{14}$ We further generated MEFs from wild-type (WT), Atg5 KO, DKO, and TKO embryos.

To detect the activation of autophagy and to determine the mechanism of autophagic cell death, MEFs were first treated with etoposide, a DNA-damaging reagent. The activation of autophagy was indicated by the punctate distribution of greenfluorescent protein (GFP)-tagged microtubule-associated protein light chain 3 (LC3) and by the production of lipid conjugates of LC3 (LC3-II). WT, but not Atg5 KO MEFs, exhibited a significant levels of autophagy (Figures 1a-c). In DKO MEFs, the number of punctate GFP-LC3 cells and the extent of LC3-II formation were greater than those of WT MEFs (Figures 1a-c). In contrast, LC3 modifications were undetectable in TKO MEFs (Figures 1a-c). Electron microscopic (EM) analysis confirmed the presence of autophagic vacuoles in etoposide-treated WT MEFs (Figures 1d and e 'WT'). In DKO MEFs, the extent of autophagy was largely increased as judged from the bigger size and the increased number of autolysosomes (Figures 1d and e 'DKO'), which is consistent with the LC3 modifications (Figures 1a-c). In TKO MEFs, we only observed a few small autophagic vacuoles (Figures 1d and e 'TKO'). The increase and decrease of autophagy in DKO MEFs and TKO MEFs, respectively, were also verified functionally using a long-lived protein degradation assay (Figure 1f). Furthermore, consistent results were obtained using an independent set of DKO and TKO MEFs (Supplementary Fig. 1a). Note that although we recently identified an Atg5-independent autophagic pathway, ${ }^{7}$ TKO MEFs, unlike Atg5 KO MEFs, exhibited limited autophagy (Figure 1d 'TKO' and Supplementary Fig. 1b and C), suggesting that Atg5-independent alternative autophagy was largely suppressed in the absence of Bax and Bak expression.

Viability of TKO and DKO MEFs. Because etoposide strongly induced autophagy in DKO MEFs, whereas the induction was greatly decreased in TKO MEFs, we reasoned that if there were more viable TKO MEFs compared with DKO MEFs, Atg5 likely contributed to etoposide-induced cell death. Therefore, we treated MEFs with etoposide and determined their viability. The viability of WT MEFs rapidly decreased, as assessed using the CellTiter-Blue (CTB) assay (Figure 2a). Morphologically, cells were rounded and detached from the culture dish $12 \mathrm{~h}$ after etoposide treatment (Figure 2b). Cell death was suppressed by zVAD-fmk, a broad caspase inhibitor, indicating that apoptosis accounts for the death of these cells (Supplementary Fig. 2a).

DKO MEFs were resistant to apoptosis such that zVAD-fmk did not affect the level of cell death (Supplementary Fig. 2b); however, etoposide still decreased their viability (Figure 2a). Morphological alteration and detachment began from $12 \mathrm{~h}$ (Figure 2b), when greater than $60 \%$ of the cells contained GFP-LC3 puncta (Figure 1b). TKO MEFs were more resistant to death (Figure 2a), and hence their detachment occurred at a later time point (Figure $2 b$ ). The higher viability of TKO MEFs compared with DKO MEFs was confirmed by cell growth (Figure 2c) and colony formation assays (Figure 2d). Similar results were obtained using an independent set of MEFs (Supplementary Fig. 2c) and when MEFs were treated with staurosporine (STS), a pan-kinase inhibitor (Figure 2e). Therefore, TKO MEFs were more resistant to cell death induced by etoposide and STS compared with DKO MEFs.

We obtained consistent results upon the silencing of Atg5 expression in DKO MEFs, indicating the involvement of Atg5 in the death of DKO MEFs (Figures $2 f$ and g, and Supplementary Fig. 2d). Because the major function of Atg5 is to regulate autophagy, we reasoned that autophagy contributed to the stress-induced death of DKO MEFs. This assumption was supported by the findings that the etoposide-induced cell death of DKO MEFs was suppressed to a level similar to that of TKO MEFs when cultures were exposed to the inhibitor of autophagy 3-methyladenine (3-MA) (Figure 2h), which did not inhibit the death of TKO MEFs (Figure $2 \mathrm{~h}$ ). Consistent results were obtained when autophagic protein degradation was blocked by lysosomal inhibitors (Supplementary Fig. 2e). Furthermore, silencing of the expression of Beclin-1 (Supplementary Fig. 2f, g), which is an essential molecule for autophagy, inhibited etoposide-induced death of DKO MEFs, but not TKO MEFs (Figures 2i and j). These data indicated that Atg5/Beclin-1-dependent autophagy is required for the stress-induced death of DKO MEFs.

We further examined the possible involvement of other modes of death in DKO and TKO MEFs. These MEFs were not rescued by nec-1 (a necroptosis inhibitor), ${ }^{15}$ ouabain (a Na/K ATPase inhibitor that suppresses autosis), ${ }^{16}$ cyclosporine A (a Cyclophilin D inhibitor that suppresses mitochondria membrane permeability transition-mediated necrosis), ${ }^{17}$ and DPQ (a PARP inhibitor that suppresses PARP-dependent necrosis) ${ }^{18}$ (Supplementary Fig. 3a, b). Furthermore, lysosomal membrane permeabilization (LMP)-mediated cell death ${ }^{19}$ was not observed because acridine-orange staining (an LMP marker) revealed that puncta formation of acidic compartments was maintained even after etoposide treatment (Supplementary Fig. 3c). Taken together, these data indicate that autophagic cell death, but not apoptosis, necroptosis, autosis, PARP-dependent necrosis, MPT-mediated necrosis, or LMP-mediated cell death, is involved in the stress-induced death of DKO MEFs.

Morphology of etoposide-treated TKO MEFs. Although TKO MEFs were resistant to apoptosis and autophagic cell death, they still lost their viability when exposed to etoposide. Therefore, to gain some insight into the mechanism of this 
death, we performed comparative EM analysis of DKO and TKO MEFs. The most prominent difference observed was hyperactivated autophagy in DKO MEFs and its absence in
TKO MEFs (Figures 3a and b). Furthermore, the nuclear membranes of DKO MEFs were intact (Figure 3a), whereas those of TKO MEFs were severely distorted and disrupted a

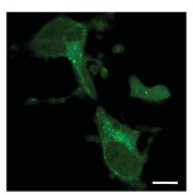

DKO

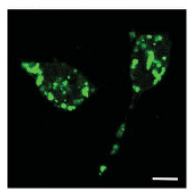

$\triangle \mathrm{ATG} 5$

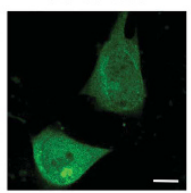

TKO

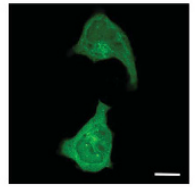

\section{b}

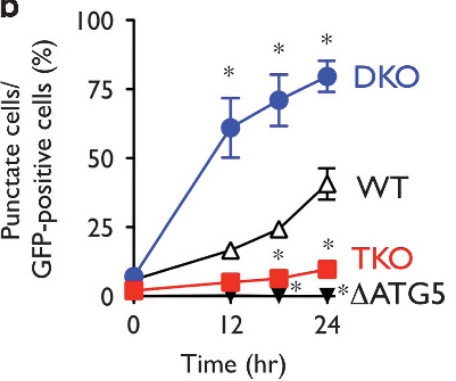

C

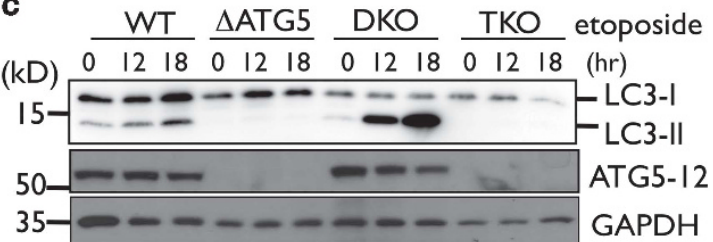

d
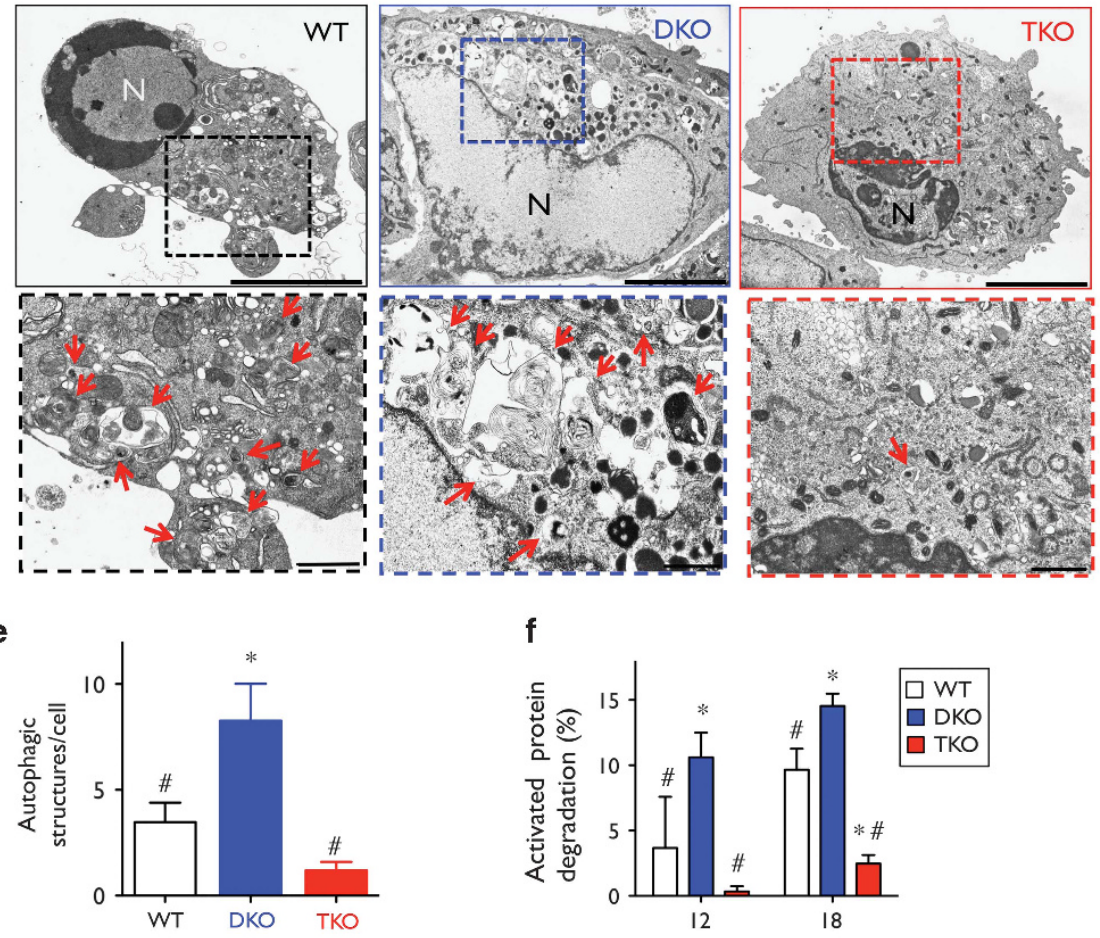

f

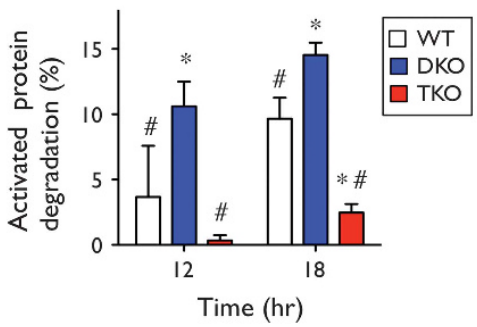

Figure 1 Lack of autophagy in Atg5/Bax/Bak TKO MEFs treated with etoposide. (a,b) MEFs transfected with GFP-LC3 were treated with $20 \mu \mathrm{M}$ etoposide for the indicated times and then examined using fluorescence microscopy. Representative images acquired at $16 \mathrm{~h}$ are shown in (a). Scale bars $=10 \mu \mathrm{m}$. (b) The population of cells with GFPLC3 puncta was calculated. Four independent experiments were performed for each type of MEF, and data represent the mean \pm S.D. ${ }^{*} P<0.05$ versus the value of WT. (c-e) MEFs were treated with $20 \mu \mathrm{M}$ etoposide for the indicated times. In panel (c), cell lysates were subjected to immunoblot analysis using antibodies against LC3, Atg5, or GAPDH (as a control). (d) (top row) Representative electron micrographs of the indicated MEFs treated with etoposide for $16 \mathrm{~h}$ (WT) and $24 \mathrm{~h}$ (DKO and TKO). 'N' indicates the nucleus. Scale bars $=5 \mu \mathrm{m}$. (bottom row) Photographs are magnified images of the areas within the dotted squares in the top row. Arrows indicate autophagosomes/autolysosomes. Scale bar $=1 \mu \mathrm{m}$. (e) The number of autophagic structures per cell was determined from the EM images. Data are shown as the mean+S.D. WT, $n=14 ;$ DKO, $n=15 ;$ TKO, $n=22$. (f) Induction of long-lived protein degradation by etoposide. WT MEFs (white columns), DKO MEFs (blue columns), and TKO MEFs (red columns) were treated with $10 \mu \mathrm{M}$ etoposide for the indicated times in the presence of $100 \mu \mathrm{M} \mathrm{zVAD}$-fmk, and the turnover of long-lived proteins was measured. Values were obtained by subtraction of basal protein degradation as follows: (\% protein degradation in etoposide-treated cells) - (\% protein degradation in healthy cells). We performed four independent experiments using each type of MEFs. Data are shown as the mean+S.D. In (e,f), ${ }^{*} P<0.05$ versus the value of $W T ;{ }^{\#} P<0.05$ versus the value of DKO 
(Figures $3 c$ and d). Staining of the nuclear membrane confirmed this distortion (Figure $3 \mathrm{e}$ ). The disruption of the nuclear membrane was evident because some mitochondria were observed within the nuclear matrix surrounded by the nuclear envelope, which was confirmed by the close localization of the nucleolonema (Figure 3d). Simple a

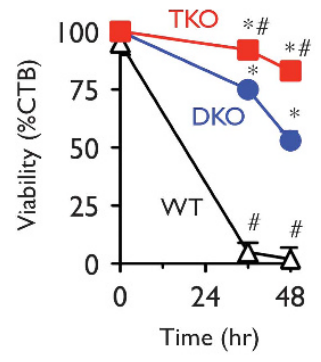

C
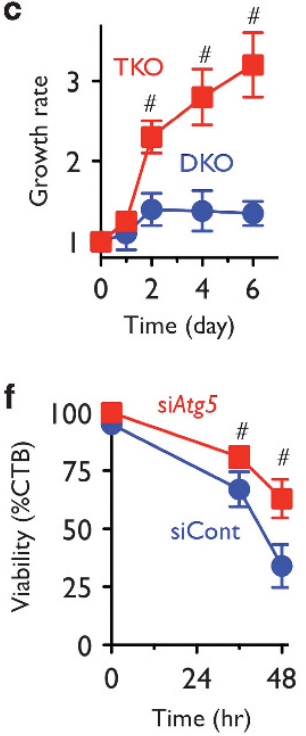

b

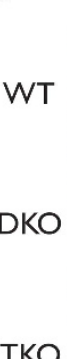

$0 \mathrm{hr}$
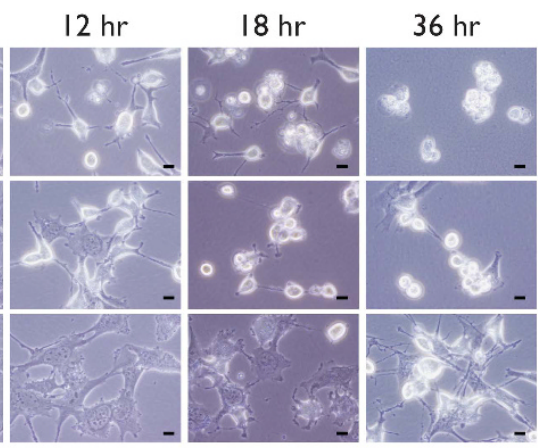

d

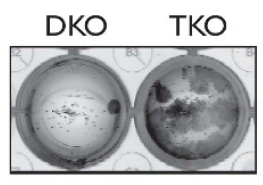

e
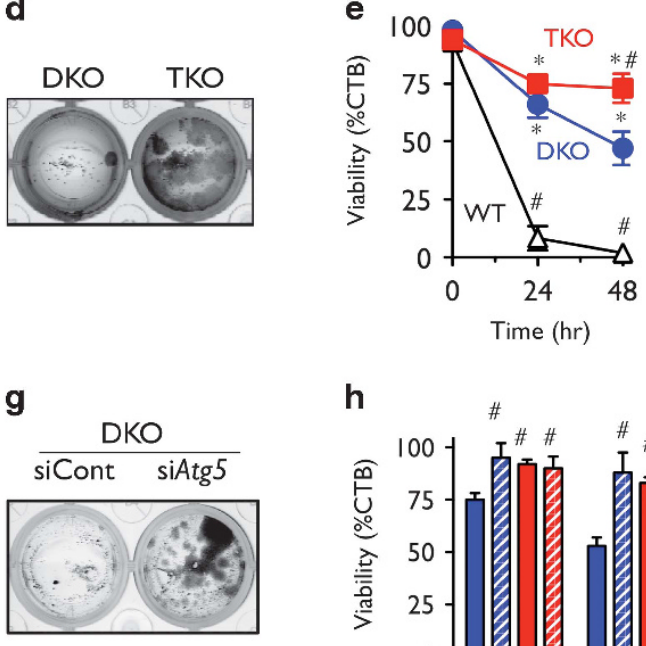

h

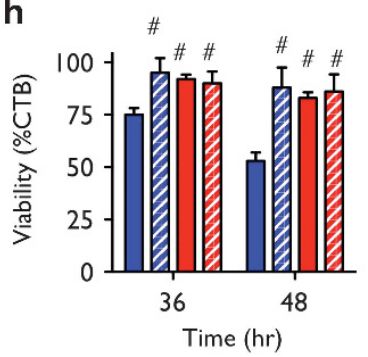

i
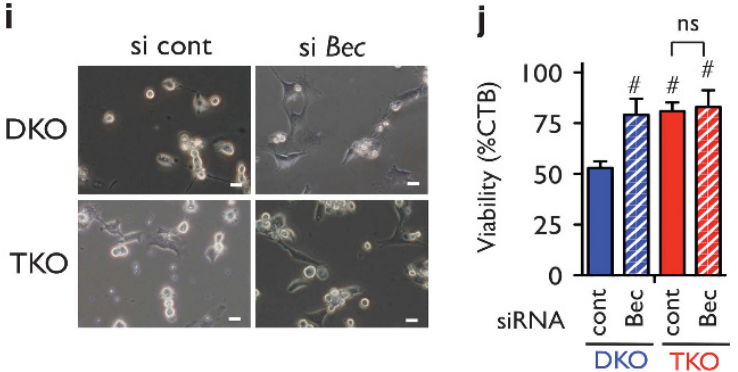

Figure 2 Viability of Atg5/Bax/Bak TKO and Bax/Bak DKO MEFs treated with etoposide and STS. (a) WT, DKO, and TKO MEFs were treated with $20 \mu$ M etoposide for the indicated times, and cell viability was assessed using the CTB assay. (b) MEFs treated with etoposide $(20 \mu \mathrm{M})$ were examined using phase-contrast microscopy. Scale bar $=10 \mu \mathrm{m}$. (c) DKO and TKO MEFs were treated with $20 \mu \mathrm{M}$ etoposide for $24 \mathrm{~h}$, and $5 \times 10^{3}$ cells were seeded in normal medium, and the number of viable cells was measured on the indicated days using the CTB assay. (d) DKO and TKO MEFs were treated with $20 \mu \mathrm{M}$ etoposide for $24 \mathrm{~h}$ and $2 \times 10^{3}$ cells were seeded in normal medium, cultured for 1 week, and the images of the colonies formed were acquired. (e) WT, DKO, and TKO MEFs were treated with $1 \mu \mathrm{M} \mathrm{STS}$ for the indicated times, and cell viability was assessed using the CTB assay. (f) Atg5-silenced DKO and control DKO MEFs were treated with $20 \mu \mathrm{M}$ etoposide for the indicated times, and cell viability was assessed using the CTB assay. (g) Atg5-silenced DKO MEFs and control DKO MEFs were treated with $20 \mu \mathrm{M}$ etoposide for $24 \mathrm{~h}$ and $2 \times 10^{3}$ cells were seeded in normal medium, cultured for 1 week, and the images of the colonies formed were acquired. (h) DKO (blue) and TKO (red) MEFs were treated with $20 \mu \mathrm{M}$ etoposide in the presence (striped columns) or absence (filled columns) of $10 \mathrm{mM}$ 3-MA for the indicated periods, and cell viability was assessed by the CTB assay. (i, j) DKO and TKO MEFs were silenced with Beclin-1 siRNA (si Bec) or control siRNA (si cont) for $24 \mathrm{~h}$ and treated with $20 \mu \mathrm{M}$ etoposide. MEFs were examined using phase-contrast microscopy. Representative images acquired at $24 \mathrm{~h}$ are shown in (i). Scale bars $=20 \mu \mathrm{m}$. (j) Cell viability was assessed at 48 h using the CTB assay. In $(a, c, e)$, data represent the mean \pm S.D. ${ }^{*} P<0.05$ versus the value of WT; ${ }^{\#} P<0.05$ versus the value of DKO. In (f), data represent the mean \pm S.D. $\# P<0.05$ versus the value of siControl DKO. In (h,j), data represent the mean+S.D. ${ }^{\#} P<0.05$ versus the value of control DKO. 'ns' indicates no significant difference. Each experiment was performed four independent times 
a

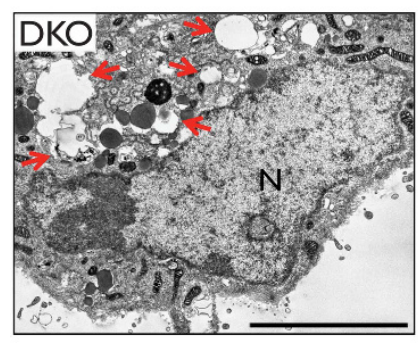

e

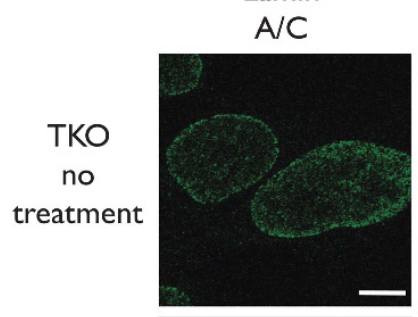

TKO

Etoposide $48 \mathrm{hr}$

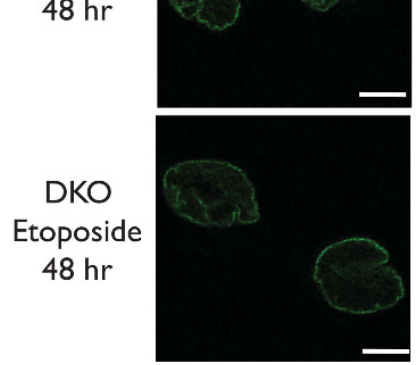

b

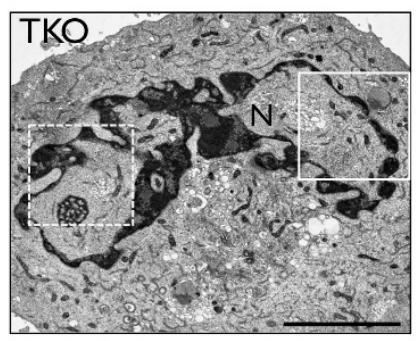

c

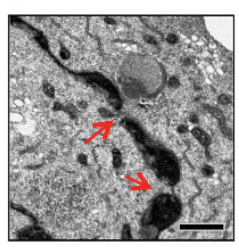

d

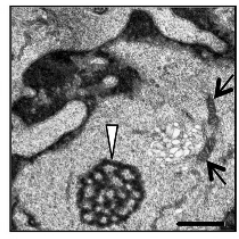

DAPI

merge
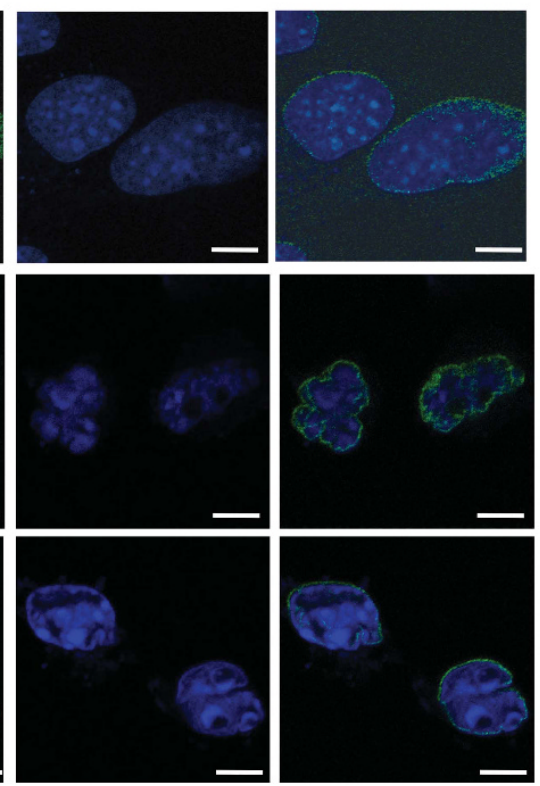

Figure 3 Morphology of Atg5/Bax/BakTKO MEFs treated with etoposide. (a-d) Representative electron micrographs of DKO and TKO MEFs treated with etoposide (20 $\mu$ M) for $24 \mathrm{~h}$. Numerous autophagosomes/autolysosomes (arrows) were observed in DKO MEFs (a) but not in TKO MEFs (b). Magnified images of the areas surrounded by a dotted box and a white box in (b) are shown in (c,d), respectively. In (c), nuclear membrane distortion (arrows) is evident. (d) Colocalization of nucleolonema (arrowhead) and mitochondria (arrows). Scale bar $=5 \mu \mathrm{m}(\mathbf{a}, \mathbf{b})$ and $1 \mu \mathrm{m}(\mathbf{c}, \mathbf{d})$. ' $N$ ' indicates the nucleus. (e) Representative laminA/C-immunofluorescence images of MEFs treated with or without etoposide for $48 \mathrm{~h}$. DNA was counterstained with DAPI. Scale bar $=5 \mu \mathrm{m}$

deformation of the nuclear membrane does not explain these observations because nuclear membrane normally separate the mitochondria and nuclear compartment. These observations are consistent with the effects of DNA-damaging agents reported by others. ${ }^{20}$

Extensive attachment of heterochromatin to the nuclear membrane of TKO MEFs was observed (Figures $1 d$ and $3 b$ ). This morphology was different from that of apoptotic cells, in which the heterochromatin was not tightly associated with the nuclear membrane. Other organelles appeared undamaged (Supplementary Fig. 4). The population of cells with abnormal nuclei was similar compared with that of dead TKO cells (Figure 2a). Therefore, although we do not have any evidence that the death of TKO MEFs can be attributed to the disruption of the structure of the nucleus, we consider that this is one reasonable mechanism of TKO cell death.
Death of DKO and TKO thymocytes. We next examined whether primary cells undergo Atg5-dependent cell death. For this purpose, we isolated thymocytes from Bak KO, DKO, and TKO embryos. Note that Bak KO mice do not demonstrate any abnormalities because of the presence of Bax. We first analyzed thymocyte development using the T-cell markers CD4 and CD8. Although the number of singlepositive thymocytes increases in adult DKO mice, ${ }^{21}$ most thymocytes from embryos of each genotype were uniformly $\mathrm{CD}^{+} \mathrm{CD}^{+}$(Supplementary Fig. 5a).

We next determined the cellular response to STS. Bak KO thymocytes showed a rapid loss of viability (Figure 4a). Compared with BakKO thymocytes, DKO thymocytes showed less cell death, and TKO thymocytes showed even less cell death (Figure 4a). Consistent results were obtained when cells were treated with different dose of STS (Supplementary 

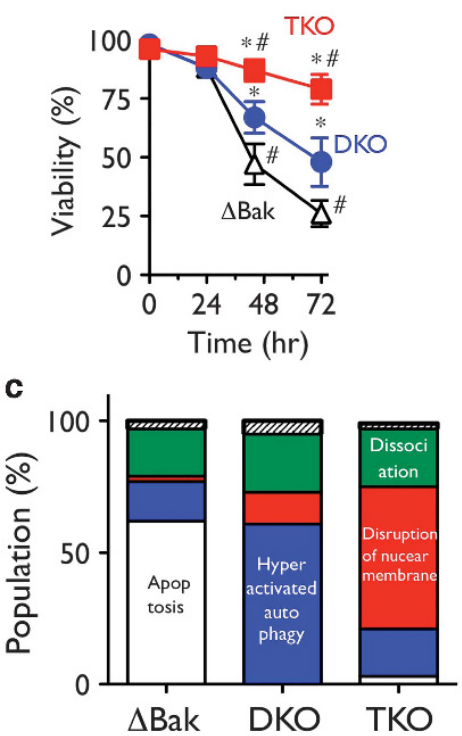

d

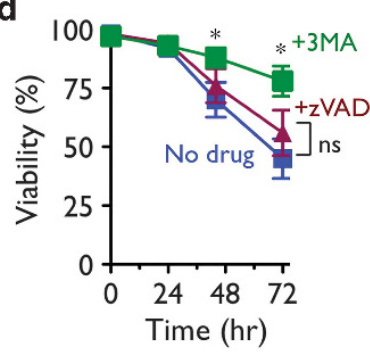

f

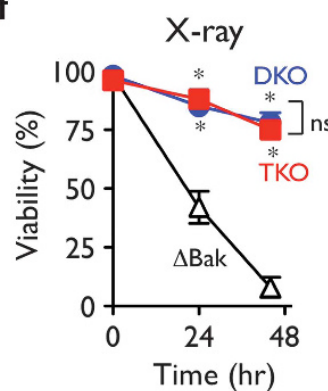

b

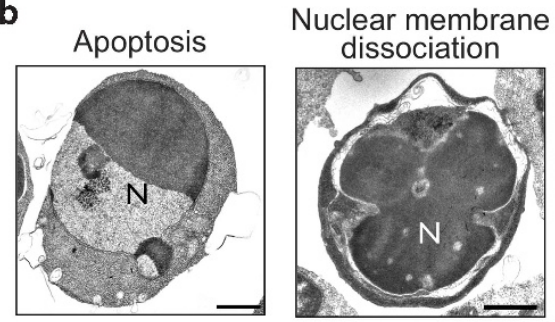

Hyperactivated autophagy
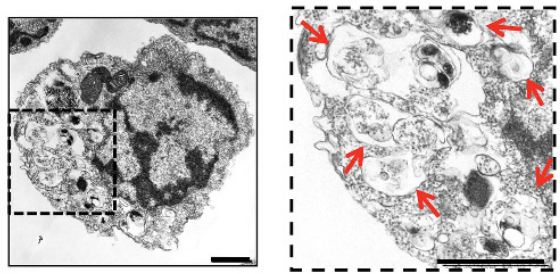

Nuclear membrane disruption
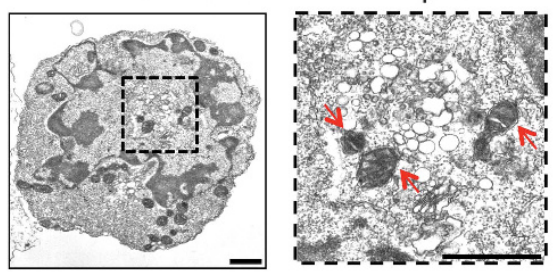

e
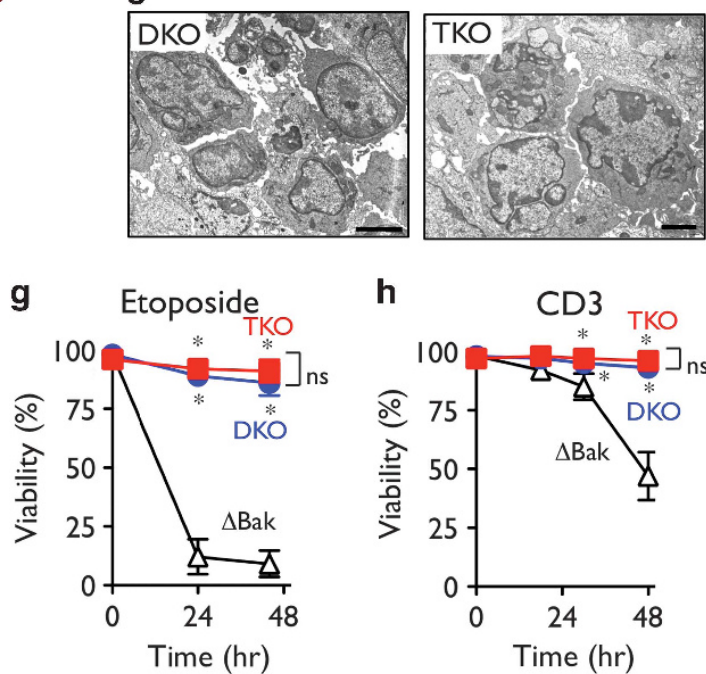

Figure 4 Viability of Atg5/Bax/BakTKO thymocytes and Bax/BakDKO thymocytes. (a) Thymocytes were treated with STS $(0.5 \mu \mathrm{M})$, and cell death was determined using the $\mathrm{Pl}$ assay at the indicated times. (b,c) Morphology of thymocytes treated with STS $(0.5 \mu \mathrm{M})$ for $12 \mathrm{~h}$. Representative electron micrographs are shown in (b). Images of 'Apoptosis' and 'Nuclear membrane dissociation' were obtained from Bak KO and DKO thymocytes, respectively. ' $\mathrm{N}$ ' indicates nuclei. Images of 'hyperactivated autophagy' were obtained from DKO thymocytes. Magnified image of the area within the square is shown on the right. Arrows indicate autophagic vacuoles. Images of 'Nuclear membrane disruption' were from TKO thymocytes. Magnified image of the area within the square is shown on the right. Arrows indicate mitochondria inside the nucleus. Scale bars $=0.5 \mu \mathrm{m}$ (magnified images) and $1 \mu \mathrm{m}$ (other images). The population of cells exhibiting each of the morphologies shown in (b) is presented in (c). 'Apoptosis,' 'Hyperactivated autophagy,' 'Nuclear membrane disruption,' and 'Nuclear membrane dissociation' are indicated in white, blue, red, and green, respectively. (d) Death of STS-treated DKO thymocytes is suppressed by 3-MA. DKO thymocytes were treated with $0.5 \mu \mathrm{M} \mathrm{STS}$ together with $5 \mathrm{mM}$ 3-MA (green symbols), $100 \mu \mathrm{M} z$-VAD-fmk (purple symbols), or without the addition of any reagent (blue symbols) for the indicated times, and cell viability was assessed using the PI assay. (e-h) Response of DKO and TKO thymocytes to X-ray irradiation, etoposide, and an antiCD3 antibody. Bak KO, DKO, and TKO thymocytes were treated with X-ray irradiation (5 Gy) (e,f), $1 \mu \mathrm{M}$ etoposide (g), or an anti-CD3 antibody (1 mg/ml) (h). (e) Representative electron micrographs of thymocytes treated with X-ray irradiation. Autophagic structures were not observed in DKO thymocytes and TKO thymocytes. Scale bars $=2 \mu \mathrm{m}$. (f-h) Cell viability was assessed using the PI assay at the indicated times. In $(\mathbf{a}, \mathbf{d}, \mathbf{f}-\mathbf{h})$, all of the experiments were performed using four independent thymi. Data represent the mean \pm SD. $(\mathbf{a}, \mathbf{f}-\mathbf{h}){ }^{*} P<0.05$ versus the value of $\Delta$ Bak; ${ }^{\#} P<0.05$ versus the value of $D K O$ thymocytes. (d) ${ }^{*} P<0.05$ versus the value of no added drug. 'ns' indicates no significant difference 
Fig. 5b). To investigate the mechanism of cell death, we performed EM observation of the dead thymocytes of each genotype. We classified the cells according to their morphological characteristics as follows: apoptotic (pyknotic cells with condensed chromatin), dissociation of outer and inner nuclear membranes, hyperactivated autophagy (resembling etoposide-treated DKO MEFs (Figure 3a)), and disrupted nuclear membrane (resembling etoposide-treated TKO MEFs (Figure 3b)) (Figure 4b). Among the four morphologically distinguishable features of dead cells, apoptotic cells predominated in Bak KO thymocytes (Figure 4c); this was confirmed by the inhibition of cell death by zVAD-fmk (Supplementary Fig. 5c). The death of most DKO thymocytes was characterized by hyperactivated autophagy (Figure 4c), which was supported by the induction of LC3-II (Supplementary Fig. 5d). Suppression of DKO thymocyte death by 3-MA but not by z-VAD-fmk (Figure 4d) suggested that the induction of autophagy was the cause of cell death. The death of TKO thymocytes was characterized by notable disruption of the nuclear membrane, and the number of autophagic cells a
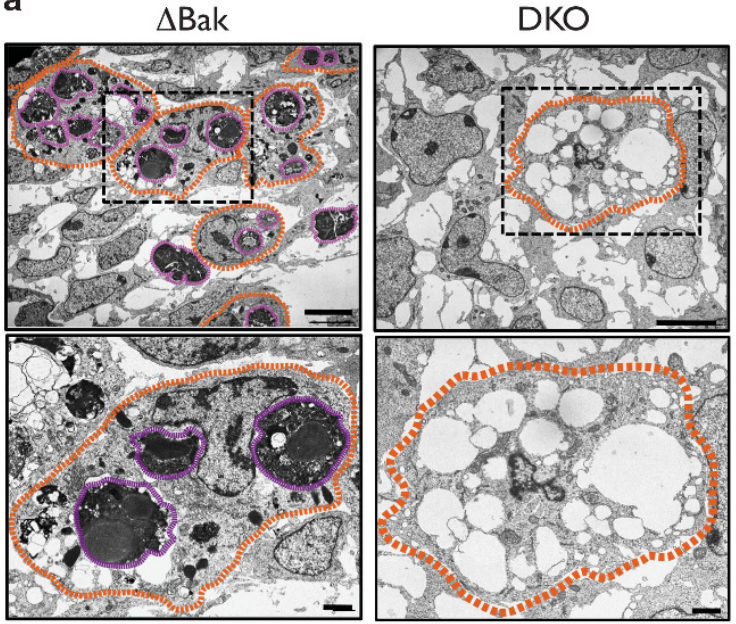

TKO

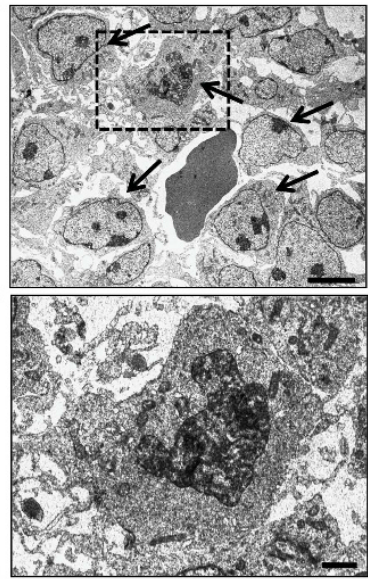

d
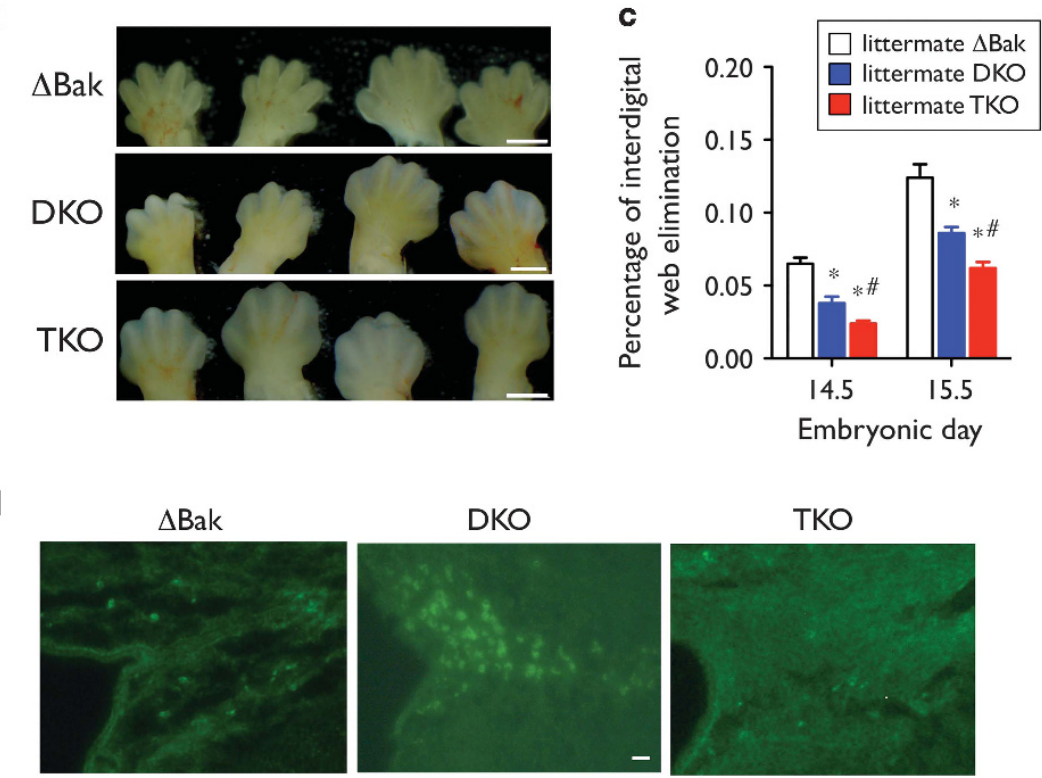

Figure 5 Delay of interdigital web disappearance in Atg5/Bax/Bak TKO embryos and Bax/Bak DKO embryos. (a) (Top row) Representative electron micrographs of the interdigital web of the indicated embryos on E14.5. Scale bars $=5 \mu \mathrm{m}$. (Bottom row) Magnified images of the areas enclosed in boxes in the top row. Scale bars $=1 \mu \mathrm{m}$. In Bak $\mathrm{KO}$ embryos, numerous apoptotic nuclei (purple circles) are engulfed by macrophages (orange circles). In DKO embryos, cells with numerous autophagic vacuoles containing digested substrates are evident (orange circle). Macrophages were not observed in the interdigital webs. In TKO embryos, most cells died with nuclear damage (arrows). Macrophages were not observed in interdigital webs. (b) Gross appearance of the interdigital webs of the limbs of indicated embryos on E14.5. Scale bar $=1 \mathrm{~mm}$. (c) Percentages of the eliminated interdigital web area were determined on E14.5 and E15.5. The method of calculation is described in Methods and Supplementary Figure 7. The same number of littermate embryos were examined for each genotype. White, blue, and red columns indicate BakKO, DKO, and TKO embryos, respectively; $n=48$ limbs (E14.5) and 36 limbs (E15.5). ${ }^{*} P<0.05$ versus Bak KO; ${ }^{\#} P<0.05$ versus DKO. (d) The presence of autophagic cells in the interdigital webs of DKO embryos. Interdigital webs from the indicated littermate embryos were frozen, and autophagic cells were visualized using immunofluorescence staining with an anti-LC3-II antibody. Representative images are shown. Autophagic cells contain LC3 puncta. Scale bar $=50 \mu \mathrm{m}$ 
was reduced compared with DKO thymocytes. These results suggest that major types of cell death in Bak KO, DKO, and TKO thymocytes were apoptosis, cell death with hyperactivated autophagy, and cell death with nuclear membrane disruption, respectively. Because the majority of DKO thymocytes died with autophagic characteristics (Figure 4c) and autophagy-resistant TKO thymocytes were more viable than DKO thymocytes (Figure 4a), we conclude that autophagy contributes to the cell death induced by STS.

Unlike STS treatment, X-ray irradiation induced only a small level of autophagy in DKO thymocytes (equivalent to that of TKO thymocytes) (Figure 4e) and weakly induced cell death to an equivalent extent to that of TKO thymocytes (Figure 4f). Similar results were obtained when DKO and TKO thymocytes were exposed to etoposide and an anti-CD3 antibody (Figures $4 \mathrm{~g}$ and $\mathrm{h}$ ), indicating that the mode of cell death was stimulus-dependent.

Autophagy-dependent PCD in the interdigital web. To determine whether Atg5-dependent cell death is involved in embryonic development, we generated DKOs and TKOs from mice on a C57BL/6J genetic background by repeated backcrossing, because several cell death-associated phenotypes are affected by genetic background. ${ }^{22}$

The loss of interdigital cells represents an example of PCD that is mediated by apoptosis. ${ }^{23}$ We observed many TUNELpositive cells in the interdigits of Bak $\mathrm{KO}$ embryos (Supplementary Fig. 6a and b). EM observation confirmed the presence of apoptotic nuclei (Figure 5a), many of which were engulfed by macrophages (Figure 5a). However, even in Apaf1 or Caspase9 KO mice, which are resistant to apoptosis, disappearance of the interdigital web occurs with some delay. We also observed this delay in Bax/Bak DKO embryos (Figure 5b,Supplementary Fig. 6c). Quantitative analysis of the area of elimination of the interdigital web per palm area (see Methods and Supplementary Fig. 7) confirmed the slower removal of the interdigital web of DKO embryos than Bak KO embryos at E14.5 and E15.5 (Figure 5c). Note that we analyzed interdigital web clearance only using littermate Bak $\mathrm{KO}$, DKO, and TKO embryos, because accurate estimation of the impregnation times of mice is difficult, and a difference of half a day results in a large difference at that stage. To determine the mechanism of disappearance of the interdigital web from DKO embryos, we performed EM observation and found that the majority of interdigital web cells contained a number of autolysosomes (Figure 5a). Moreover, some cells appeared to be destroyed because they were filled with autolysosomes, as shown in Figure $5 \mathrm{a}$. These cells resemble with etoposide-treated DKO MEFs and STS-treated DKO thymocytes, suggesting autophagic cell death. The presence of numerous LC3-positive puncta (Figure 5d) and the absence of TUNEL-positive cells (Supplementary Fig. 6b) among the interdigital web cells support this conclusion.

In TKO embryos, loss of the interdigital web was further delayed in the absence of autophagy (Figures $5 b$ and $c$, Supplementary Fig. 6c), suggesting that Atg5-dependent cell death contributed to the elimination of interdigital webs. Interdigital cells of TKO embryos contained only a few autophagic vacuoles but had patchy chromatin structure and disrupted nuclear membranes (Figure 5a) similar to those of etoposide-treated TKO MEFs and STS-treated TKO thymocytes. These data suggest that autophagic cell death plays a crucial role in the embryonic development of apoptosisresistant mice.

Apoptotic cells are quickly removed by macrophages via a mechanism that is dependent on the exposure of phosphatidyl serine (PS) on the cell membrane, ${ }^{24}$ as was observed in tissue sections of the interdigital webs of Bak KO embryos (Figure 5a). However, we did not observe macrophages in the interdigital webs of DKO and TKO embryos (Figure 5a), probably because PS exposure was not actively induced in dying DKO and TKO cells. Furthermore, PS exposure was delayed significantly in etoposide-treated DKO and TKO MEFs (Supplementary Fig. $8 a$ and b), and these cells were not engulfed by macrophages (Supplementary Fig. 8c and d). In the interdigital webs of DKO and TKO embryos, dying cells should be eliminated by shedding, and not by macrophage engulfment.

Abnormal embryonic death and brain development of
Atg5/Bax/Bak TKO embryos. Finally, we examined the other phenotypes of DKO and TKO embryos. When we counted the number of viable embryos on various embryonic day, the population of TKO embryos, but not DKO embryos, was $\sim 50 \%$ lower than the expected Mendelian rate (Figure 6a). Consistently, we observed dead TKO embryos on each embryonic day (Figure 6b). Because a large number of dead embryos were observed on E13.5 and then gradually decreased, and as all the dead embryos exhibited organogenesis failure with some of them beginning to disintegrate (Figure 6c). TKO embryos seemed to die before E13.5 and then be reabsorbed by the uterus. These data indicated that DKO embryos developed normally, whereas TKO embryos are prone to embryonic death.

In addition to interdigital web elimination and embryonic death, we also observed exencephaly in some, but not all, TKO embryos (Figure 6d). Exencephaly is a common phenotype of apoptosis-deficient mice, ${ }^{23}$ which develops because of incomplete neural tube closure caused by a dysregulation of apoptosis, ${ }^{25}$ and largely depends on the mouse strain. For example, exencephaly occurs in the 129 strain but only rarely in the $\mathrm{C} 57 \mathrm{BL} / 6 \mathrm{~J}$ strain. ${ }^{22}$ We consistently observed exencephaly in only $3.6 \%$ of DKO embryos on a C57BL/6J background compared with $0.6 \%$ of WT embryos, and this difference was not statistically significant (Figure 6e). In contrast, we observed exencephaly in $\sim 40 \%$ of TKO embryos on a C57BL/6J background (Figure 6e), which showed a statistically significant difference from DKO embryos. The morphology is similar to the exencephaly observed in Apaf1 KO embryos on a 129 background. $^{26}$ These data, taken together with the absence of detectable exencephaly in Atg5 KO embryos, suggest that brain development in DKO embryos proceeds normally, probably by the compensation of autophagic cell death for the apoptosis deficiency on a C57BL/6J background. Therefore, a simultaneous loss of apoptosis and autophagic cell death may increase the rate of exencephaly (see Discussion). Taken together, we suggest that in apoptosis-deficient mice, autophagic cell death partly mediates developmental cell death by compensating for the deficiency in apoptosis. 
Number of viable embryos (birth rate)

\begin{tabular}{|c|c|c|c|c|c|}
\hline Embryonic day & 13.5 & 14.5 & 16.5 & 18.5 & Expected rate \\
\hline Atg5 KO & $105(19.0 \%)$ & $38(19.7 \%)$ & $27(19.6 \%)$ & $59(18.3 \%)$ & $19 \%$ \\
\hline DKO & $111(20.1 \%)$ & $42(21.8 \%)$ & $30(21.7 \%)$ & $71(22.0 \%)$ & $19 \%$ \\
\hline TKO & $19(3.4 \%)$ & $7(3.6 \%)$ & $4(2.9 \%)$ & $8(2.5 \%)$ & $6 \%$ \\
\hline others & $318(57.5 \%)$ & $106(54.9 \%)$ & $77(55.8 \%)$ & $185(57.3 \%)$ & $56 \%$ \\
\hline Total number of pups & 553 & 193 & 138 & 323 & $100 \%$ \\
\hline
\end{tabular}

b

Number of dead embryos/ total embryos

\begin{tabular}{|c|c|c|c|c|}
\hline Embryonic day & 13.5 & 14.5 & 16.5 & 18.5 \\
\hline Atg5 KO & $0 / 105$ & $0 / 38$ & $0 / 27$ & $1 / 60(1.7 \%)$ \\
\hline DKO & $0 / 111$ & $0 / 42$ & $0 / 30$ & $0 / 71$ \\
\hline TKO & $11 / 30(36.7 \%)$ & $2 / 8(25.0 \%)$ & $1 / 5(20.0 \%)$ & $1 / 10(10.0 \%)$ \\
\hline others & $0 / 318$ & $2 / 108(1.9 \%)$ & $0 / 77$ & $2 / 187(1.1 \%)$ \\
\hline
\end{tabular}

C

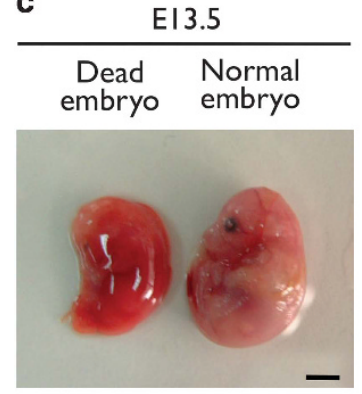

d E.14.5

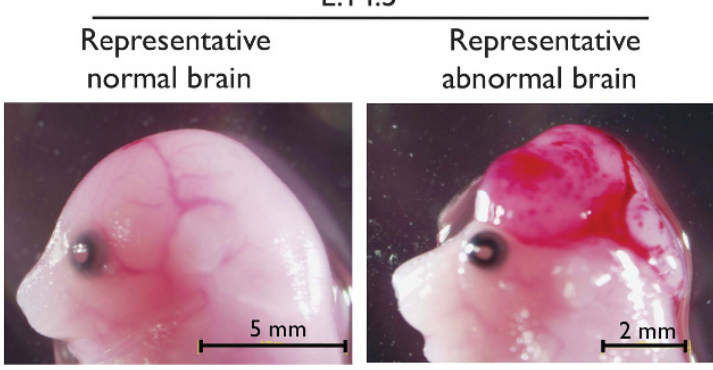

e

Number of embryos with abnormal brain/ viable embryos

\begin{tabular}{|c|c|c|c|c|}
\hline Embryonic day & 13.5 & 14.5 & 16.5 & 18.5 \\
\hline Atg5 KO & $0 / 105(0 \%)$ & $0 / 38(0 \%)$ & $0 / 27(0 \%)$ & $0 / 59(0 \%)$ \\
\hline DKO & $4 / 111(3.6 \%)$ & $0 / 42(0 \%)$ & $0 / 30(0 \%)$ & $0 / 71(0 \%)$ \\
\hline TKO & $8 / 19(42.1 \%)$ & $3 / 7(42.9 \%)$ & $2 / 4(50.0 \%)$ & $2 / 8(25.0 \%)$ \\
\hline others & $2 / 318(0.6 \%)$ & $0 / 106(0 \%)$ & $0 / 77(0 \%)$ & $0 / 185(0 \%)$ \\
\hline
\end{tabular}

Figure 6 Death and abnormal brain development in TKO embryos on a B6 background. (a,b) The tables show the population of viable embryos (a) and dead embryos (b) on the indicated embryonic days. In (a), 'Expected rate' indicates the Mendelian ratio. Yellow rows indicate 'TKO'. (c) A representative dead TKO embryo is shown together with a normal embryo on E13.5. Scale bar $=2 \mathrm{~mm}$. (d) Representative photographs showing brain malformation in TKO embryos with a normal embryo in a C57BL/6 background. (e) The table indicates the population of embryos with an abnormal brain. Yellow row indicates 'TKO'

\section{Discussion}

For many years, physiological cell death was thought to be caused by apoptosis. However, certain types of nonapoptotic cell death were discovered after the central mechanism of apoptosis was defined. Because apoptosis-deficient embryos develop nearly normally until birth, nonapoptotic cell death must play crucial roles in their development and may compensate for apoptosis in apoptosis-deficient mice or play an intrinsic role that is required for normal development.

Autophagy-dependent death plays a role in the development of the larval salivary glands of Drosophila. ${ }^{4,10}$ Although a similar type of machinery is expected to exist in other organisms, to date, definitive evidence to suggest the existence of autophagy-dependent cell death in vivo in mammals is lacking. For example, Atg5 and Atg7 KO mouse embryos do not show detectable abnormalities during development. However, the Atg5/Atg7-independent autophagy pathway that we recently discovered ${ }^{7}$ may compensate for the developmental abnormalities caused by Atg5/Atg7 deficiency. Alternatively, deficiency of autophagy-dependent death might be compensated by apoptosis. Likewise, autophagic cell death may compensate for a deficiency or dysregulation of apoptosis. Therefore, developmental abnormalities might become apparent only in organisms deficient for both apoptosis and autophagy. Therefore, in this study, we evaluated this possibility and show that Atg5 deficiency caused embryonic death and enhanced developmental abnormalities, such as excencephaly and a delay in the elimination of interdegital webs, on an apoptosis-resistant background. Among these phenotypes, interdigital web clearance requires cell loss, and hence the induction of Atg5-dependent death should be the cause of the different phenotypes observed in DKO and TKO embryos. Regarding exencephaly, this occurs by a failure of neural tube closure in early stage embryos, and not only cell death-related genes but also genes governing proliferation, differentiation, and even actin regulation, are committed to this phenotype. However, of 
the various types of exencephaly, forebrain protrusion is only observed in cell death-related gene mutants, ${ }^{27,28}$ and hence Atg5-dependent death might also be involved in the exencephaly (forebrain protrusion) in TKO embryos. These findings highlight a crucial role for autophagy-dependent death in mice, at least under conditions of apoptosis deficiency. Although recent findings show that necroptosis-deficient Rip3 KO and MlkI KO mice do not exhibit detectable developmental abnormalities, ${ }^{29,30}$ necroptosis might be involved in the compensation of apoptosis deficiency.

How do cells die by autophagic cell death? The detailed molecular mechanisms are unknown; however, our ultrastructural analysis provides some insights. First, there is a robust increase in the number and size of autophagic vacuoles in cells undergoing autophagic cell death compared with those undergoing starvation-induced autophagy. The diameter of starvation-induced autophagic vacuoles is mostly $<1 \mu \mathrm{m}$, whereas autophagic vacuoles greater than $3 \mu \mathrm{m}$ in diameter are frequently observed during autophagic cell death. Therefore, the hyperactivation of autophagy may be crucial for the induction of autophagic cell death. Ultrastructural and functional analyses of autophagic cell death indicate that most organelles, including mitochondria and the ER, appear normal. Therefore, it is unlikely that the degradation of specific organelles initiates autophagic cell death. Rather, we consider that the excessive degradation of cytosolic components by autophagy may represent the direct cause of autophagic cell death.

Taken together, the data presented here suggest an important role for autophagic cell death during the development of apoptosis-deficient mice. The role of this process in physiological contexts remains to be determined.

\section{Materials and Methods}

Generation of mutant mice. $\mathrm{Bax}^{+/-} \mathrm{Bak}^{-/}$mice were crossed with C57BL/6 mice for at least 10 generations. Atg5/Bax/Bak TKO mice were generated by breeding $\mathrm{Bax}^{+/-} \mathrm{Bak}^{-/-}$mice with $\mathrm{C} 57 \mathrm{BL} / 6 \mathrm{Atg}^{+/-}$mice. The C57BL/6 background $(>95 \%)$ was confirmed using 100 microsatellite markers. All mice were bred at the Laboratory for Recombinant Animals, Medical Research Institute, Tokyo Medical and Dental University; the Animal Facility of Osaka University Medical School; and the Animal Facility of Oriental Bioservice, Inc.

Antibodies and chemicals. An anti-Atg5 polyclonal antibody (Sigma-Aldrich Inc., St. Louis, MO, USA) was used for immunoblot assays. An anti-laminA/C (Cell Signaling Technology Inc., Danvers, MA, USA) monoclonal antibody was used for immunofluorescence assays. Anti-LC3 (NanoTools Inc.) and anti-GAPDH (Santa Cruz Biotechnology, Inc., Santa Cruz, CA, USA) monoclonal antibodies were used for immunoblot assays. The chemicals 3-MA, z-VAD-fmk, and DAPI were obtained from Sigma-Aldrich, Peptide Inc. (Ibaraki, Osaka, Japan), and Molecular Probes (Eugene, OR, USA), respectively. TUNEL Assay Kit was purchased from Chemicon International Co (Temecula, CA, USA). All other chemicals were purchased from Nacalai Tesque.

Cell culture and transfection. WT, $\mathrm{Atg}^{--}, \mathrm{Bax}^{-/} \mathrm{Bak}^{-/}$(DKO), and Atg $5^{-1} \mathrm{Bax}^{-1-} \mathrm{Bak}^{-1-}$ (TKO) MEFs were harvested from mouse embryos on E14.5 and immortalized with SV40 T antigen. ${ }^{12}$ MEFs that were passaged in the same way for over 15 passages were used. The passage number did not affect the cellular phenotype. Bak KO, DKO, and TKO primary thymocytes were harvested from the respective embryos on E18.5, as described previously. ${ }^{21}$ MEFs and thymocytes were cultured in Dulbecco's modified Eagle's medium supplemented with $2 \mathrm{mM}$ L-glutamine, $1 \mathrm{mM}$ sodium pyruvate, $0.1 \mathrm{mM}$ nonessential amino acids, $10 \mathrm{mM}$ HEPES/Na ${ }^{+}$(pH 7.4), $0.05 \mathrm{mM}$ 2-mercaptoethanol, $100 \mathrm{U} / \mathrm{ml}$ penicillin, $100 \mu \mathrm{g} / \mathrm{ml}$ streptomycin, and $10 \%$ fetal bovine serum.
In some experiments, MEFs $\left(1 \times 10^{6}\right)$ were transfected with GFP-LC3 plasmid DNA using the Amaxa electroporation system according to the supplier's protocol (Kit V, Program U-20). The transfection efficiency was $>75 \%$, as assessed by GFPfluorescent cells. MEFs $\left(1 \times 10^{6}\right)$ were also transfected with $10 \mu \mathrm{g}$ of siRNA using RNAiMax (Thermo Co.) according to the supplier's protocol. The siRNA sequences used were as follows: mouse Beclin-1 5'-UUGGUUUGGAAAGAUGCUUUA-3', mouse Atg5 5'-GAGUCAGCUAUUUGACGUU-3', and control siRNA (Dharmacon siGENOME Non-Targeting siRNA).

Staining of autophagosomes. Formation of LC3 puncta was examined using MEFs transiently transfected with $1 \mu \mathrm{g}$ of the GFP-LC3 expression plasmid. ${ }^{31}$ Cells were treated with etoposide for $24 \mathrm{~h}$ after transfection, and GFP-LC3 fluorescence was observed using a confocal fluorescence microscope (LSM 510 META, Zeiss). Mouse tissues were fixed in $4 \%$ paraformaldehyde in PBS for $4 \mathrm{~h}$ and embedded in paraffin for the preparation of $2-\mu \mathrm{m}$ sections and were subjected to TUNEL staining according to the supplier's protocol. Immunofluorescence analysis was also performed using an anti-LC3 antibody.

Western blot analysis. Whole-cell protein extracts were prepared using RIPA buffer, and protein concentration was quantified using the Lowry method (Bio-Rad, Hercules, CA, USA) according to the manufacturer's protocol. Proteins were separated by electrophoresis through $15 \%$ SDS-polyacrylamide gels and transferred to PVDF membranes. The membranes were incubated with the antibodies indicated in the figures, and the immune complexes were detected using the appropriate HRP-conjugated secondary antibodies and ECL reagent (Amersham, Little Chalfont, UK).

Assay of long-lived protein degradation. Degradation of long-lived proteins was measured as described previously. ${ }^{7}$ Briefly, cells were labeled for $20 \mathrm{~h}$ with a medium containing $0.2 \mu \mathrm{Ci} / \mathrm{ml} \mathrm{L-}\left[{ }^{14} \mathrm{C}\right]$ valine (GE Healthcare Inc.). After washing and incubation in a medium containing $10 \mathrm{mM}$ cold valine for $60 \mathrm{~min}$, the medium was replaced with fresh medium and further incubated for the indicated times. Macromolecules in the medium were then precipitated using 10\% TCA, and TCA-soluble radioactivity was measured. $\left[{ }^{14} \mathrm{C}\right]$-valine release was calculated as a percentage of the total radioactivity in the TCA-soluble supernatant. The extent of protein degradation was calculated by subtracting the $\left[{ }^{14} \mathrm{C}\right]$-valine release by untreated cells from that by starved or etoposide-treated cells.

Cell viability assay. MEFs were seeded onto 6-well dishes at $3.5 \times 10^{6}$ cells per well. After $24 \mathrm{~h}$, the cells were exposed to etoposide $(20 \mu \mathrm{M})$ or STS $(1 \mu \mathrm{M})$ in the presence or absence of 3-MA $(10 \mathrm{mM})$ or Z-VAD-fmk $(100 \mu \mathrm{M})$. After the observation of cells using phase-contrast microscopy, their viability was assessed using the CTB assay to measure the reduction of resazurin, which measures the metabolic activity of viable cells. ${ }^{12}$ Briefly, $5 \times 10^{4}$ cells were harvested, and the CTB assay reagent was added to the cells according to the supplier's protocol. The extent of dead cells was calculated as the percentage relative to the initial value at each sampling time. To measure cell proliferation, MEFs were treated with etoposide, all cells were recovered, and $5 \times 10^{3}$ cells were cultured again in standard medium in 48-well dishes. Viable cells were measured on the indicated days using the CTB assay. ${ }^{12}$ For the colony formation assay, MEFs were treated with etoposide, harvested, and $2 \times 10^{3}$ cells were seeded onto 24-well dishes containing standard medium. After 1 week, cells were stained with calcein-AM, and images were acquired using fluorescence microscopy. Primary thymocytes were seeded onto 6 -well dishes at $3.5 \times 10^{5}$ cells per well. After $24 \mathrm{~h}$, the cells were exposed to STS $(0.5 \mu \mathrm{M})$, etoposide $(1 \mu \mathrm{M})$, or an anti-CD3 antibody $(1 \mathrm{mg} / \mathrm{ml})$ in the presence or absence of 3-MA $(10 \mathrm{mM})$ or Z-VAD-fmk $(100 \mu \mathrm{M})$. Cells were harvested, and their viability was determined using PI staining.

Staining of the nuclear membrane. Cells were fixed in $4 \%$ formaldehyde, permeabilized in $0.1 \%$ Triton X-100, stained with an anti-laminA/C antibody, and incubated with an Alexa488-conjugated secondary antibody. DNA was counterstained with DAPI.

Electron microscopy. Cells were fixed with $1.5 \%$ paraformaldehyde $/ 3 \%$ glutaraldehyde in $0.1 \mathrm{M}$ phosphate buffer $\left(\mathrm{pH} 7.2\right.$ ) followed by fixation in $1 \% \mathrm{OsO}_{4}$. After dehydration, ultrathin sections were stained with uranyl acetate and lead citrate and observed using a JEM-1010 electron microscope (JEOL Co. Ltd.) at $80 \mathrm{kV}$. 
Analysis of interdigital webs. We crossed $\mathrm{Atg}^{+/-} \mathrm{Bax}^{+/-} \mathrm{Bak}^{-/-}$mice to each other, and examined only a set of Bak KO, Bax/Bak DKO, and Atg5/Bax/Bak TKO littermates for the analysis of interdigital web elimination. An embryo was judged as having a web when a web was clearly present in at least one leg. The percentage of the eliminated interdigital web area was calculated by dividing the area surrounded by the proximal points of the thumb and little finger and all of the fingertips by the area of elimination of the interdigital web. Representative calculations are shown in Supplementary Fig. 7.

Statistical analysis. Results are expressed as the mean \pm S.D. Statistical evaluation was performed using Prism (GraphPad) software. Comparisons of multiple data sets were performed using one-way ANOVA followed by the Tukey post hoc test. A $P$-value of $<0.05$ was considered to indicate a statistically significant difference between two groups. Statistical analyses of non-random associations between two categorical variables were examined using the Fisher exact test.

\section{Conflict of Interest}

The authors declare no conflict of interest.

Acknowledgements. We are grateful to $\mathrm{Dr}$ N. Mizushima for providing us with Atg $5^{+/-}$mice. This study was supported in part by Grant-in-Aid for Grant-in-Aid for Scientific Research (S) (22229002), Grant-in-Aid for Scientific Research (A) (17H01533), Grant-in-Aid for challenging Exploratory Research (16K15230), Grantin-Aid for Scientific Research on Innovative Areas (15H01554, 26110001, 26110005), Grant-in-Aid for Encouragement of Young Scientists (B) (15K19004) from the MEXT of Japan, by the Project for Cancer Research and Therapeutic Evolution (P-CREATE) and by the Project for Psychiatric and Neurological Disorders from the Japan Agency for Medical Research and development, AMED. This study was also supported by the Joint Usage/Research Program of Medical Research Institute, Tokyo Medical and Dental University, and by a grant from the Secom Science and Technology Foundation and the Takeda Science Foundation.

\section{Author contributions}

SA performed EM analyses. MT, TY, HS, YN, YM, IY, and SS performed other experiments and analyzed the results. YT and SS designed and conducted the research, and wrote the paper.

1. Clarke PG. Developmental cell death: morphological diversity and multiple mechanisms. Anat Embryol 1990; 181: 195-213.

2. Tsujimoto Y. Cell death regulation by the Bcl-2 protein family in the mitochondria. J Cell Physiol 2003; 195: 158-167.

3. Baehrecke EH. How death shapes life during development. Nat Rev Mol Cell Biol 2002; 3: 779-787.

4. Das G, Shravage BV, Baehrecke EH. Regulation and function of autophagy during cell survival and cell death. Cold Spring Harb Perspect Biol 2012; 4: a008813.

5. Xie Z, Klionsky DJ. Autophagosome formation: core machinery and adaptations. Nat Cell Biol 2007; 9: 1102-1109.

6. Mizushima N, Yoshimori T, Ohsumi Y. The role of Atg proteins in autophagosome formation. Annu Rev Cell Dev Biol 2011; 27: 107-132.

7. Nishida Y, Arakawa S, Fujitani K, Yamaguchi H, Mizuta T, Kanaseki T et al. Discovery of Atg5/Atg7-independent alternative macroautophagy. Nature 2009; 461: 654-658.
8. Levine B, Yuan J. Autophagy in cell death: an innocent convict? J Clin Invest 2005; 115: 2679-2688.

9. Shen HM, Codogno P. Autophagic cell death: Loch Ness monster or endangered species? Autophagy 2011; 7: 457-465.

10. Denton $\mathrm{D}$, Nicolson $\mathrm{S}, \mathrm{Kumar} \mathrm{S}$. Cell death by autophagy: facts and apparent artifacts. Cell Death Differ 2012; 19: 87-95.

11. Tracy K, Baehrecke EH. The role of autophagy in Drosophila metamorphosis. Curr Top Dev Biol 2013; 103: 101-125.

12. Shimizu S, Kanaseki T, Mizushima N, Mizuta T, Arakawa-Kobayashi S, Thompson CB et al. Role of Bcl-2 family proteins in a non-apoptotic programmed cell death dependent on autophagy genes. Nat Cell Biol 2004; 6: 1221-1228.

13. Eisenberg-Lerner A, Bialik S, Simon HU, Kimchi A. Life and death partners: apoptosis, autophagy and the cross-talk between them. Cell Death Differ 2009; 16: 966-975.

14. Kuma A, Hatano M, Matsui M, Yamamoto A, Nakaya H, Yoshimori T et al. The role of autophagy during the early neonatal starvation period. Nature 2004; 432: 1032-1036.

15. Degterev A, Huang Z, Boyce M, Li Y, Jagtap P, Mizushima N et al. Chemical inhibitor of nonapoptotic cell death with therapeutic potential for ischemic brain injury. Nat Chem Biol 2005; 1: 112-119.

16. Liu $Y$, Shoji-Kawata S, Sumpter RM Jr, Wei $Y$, Ginet V, Zhang $L$ et al. Autosis is a Na+, $\mathrm{K}+$-ATPase-regulated form of cell death triggered by autophagy-inducing peptides, starvation, and hypoxia-ischemia. Proc Natl Acad Sci USA 2013; 110: 20364-20371.

17. Nakagawa T, Shimizu S, Watanabe T, Yamaguchi O, Otsu K, Yamagata $\mathrm{H}$ et al. Cyclophilin $D$-dependent mitochondrial permeability transition regulates some necrotic but not apoptotic cell death. Nature 2005; 434: 652-658.

18. Zong WX, Ditsworth D, Bauer DE, Wang Z-Q, Thompson CB. Alkylating DNA damage stimulates a regulated form of necrotic cell death. Genes Dev 2004; 18: 1272-1282.

19. Boya P, Kroemer G. Lysosomal membrane permeabilization in cell death. Oncogene 2008; 27: 6434-6451.

20. Kaufmann $\mathrm{SH}$. Induction of endonucleolytic DNA cleavage in human acute myelogenous leukemia cells by etoposide, camptothecin, and other cytotoxic anticancer drugs: a cautionary note. Cancer Res 1989; 49: 5870-5878.

21. Rathmell JC, Lindsten T, Zong W-X, Cinalli RM, Thompson CB. Deficiency in Bak and Bax perturbs thymic selection and lymphoid homeostasis. Nat Immunol 2002; 3 : 932-939.

22. Houde C, Banks KG, Coulombe N, Rasper D, Grimm E, Roy S et al. Caspase-7 expanded function and intrinsic expression level underlies strain-specific brain phenotype of caspase3-null mice. J Neurosci 2004; 24: 9977-9984.

23. Lindsten T, Ross AJ, King A, Zong WX, Rathmell JC, Shiels HA et al. The combined functions of proapoptotic $\mathrm{Bcl}-2$ family members Bak and Bax are essential for normal development of multiple tissues. Mol Cell 2000; 6: 1389-1399.

24. Miyanishi M, Tada K, Koike M, Uchiyama Y, Kitamura T, Nagata S. Identification of Tim4 as a phosphatidylserine receptor. Nature 2007; 450: 435-439.

25. Yamaguchi Y, Shinotsuka N, Nonomura K, Takemoto K, Kuida K, Yosida $\mathrm{H}$ et al. Live imaging of apoptosis in a novel transgenic mouse highlights its role in neural tube closure. J Cell Biol 2011; 195: 1047-1060.

26. Yoshida H, Kong YY, Yoshida R, Elia AJ, Hakem A, Hakem R et al. Apaf1 is required for mitochondrial pathways of apoptosis and brain development. Cell 1998; 94: 739-750.

27. Harris MJ, Juriloff DM. Mouse mutants with neural tube closure defects and their role in understanding human neural tube defects. Birth Defects Res A Clin Mol Teratol 2007; 79: $187-210$.

28. Nagasaka A, Kawane $\mathrm{K}$, Yoshida $\mathrm{H}$, Nagata S. Apaf-1-independent programmed cell death in mouse development. Cell Death Differ 2010; 17: 931-941.

29. Newton K, Sun X, Dixit VM. Kinase RIP3 is dispensable for normal NF-kBs, signaling by the B-cell and T-cell receptors, tumor necrosis factor receptor 1 , and toll-like receptors 2 and 4 . Mol Cell Biol 2004; 24: 1464-1469.

30. Wu J, Huang Z, Ren J, Zhang Z, He P, Li Y et al. Mlkl knockout mice demonstrate the indispensable role of Mikl in necroptosis. Cell Res 2013; 23: 994-1006.

31. Kabeya Y, Mizushima N, Ueno T, Yamamoto A, Kirisako T, Noda T et al. LC3, a mammalian homologue of yeast Apg8p, is localized in autophagosome membranes after processing. EMBO J 2000; 19: 5720-5728.

\section{Supplementary Information accompanies this paper on Cell Death and Differentiation website (http://www.nature.com/cdd)}

